\title{
Prenatal Maternal Stress and Epigenetics: Review of the Human Research
}

\author{
Lei Cao-Lei ${ }^{1,2}$ • David P. Laplante ${ }^{2}$. Suzanne King ${ }^{1,2}$
}

Published online: 17 February 2016

(C) Springer International Publishing AG 2016

\begin{abstract}
Alterations of the uterine environment might induce long-lasting effects on offspring outcomes in later life. Epigenetics has been considered as one of the most important mechanisms involved in such long-term effects of exposure to stress in utero. In the present review, we summarize a growing body of literature focused on human research, exploring the association between fetal environmental experience and epigenetic change, especially DNA methylation. Evidence from methylation of genes associated with intrautrine adversity has shed light on how DNA methylation patterns may mediate biological processes that are involved in stress regulation. Furthermore, we report on the epigenetic findings from Project Ice Storm, a human model to study prenatal maternal stress. The conclusion of this review is that there is emerging evidence supporting the critical mediating role of epigenetics, highlighting the importance of long-term adaptation and programming through epigenetic mechanisms in response with environmental factors.
\end{abstract}

Keywords Prenatal maternal stress · Fetal programming . Epigenetics $\cdot$ DNA methylation $\cdot$ Mediating effect $\cdot$ Natural disaster

This article is part of the Topical Collection on Epigenetics

Suzanne King

suzanne.king@douglas.mcgill.ca

1 Department of Psychiatry, McGill University, Montreal, Quebec, Canada

2 Psychosocial Research Division, Douglas Hospital Research Centre, Montreal, Quebec, Canada

\section{Introduction: Prenatal Maternal Stress, Epigenetics, and Development}

Human and animal studies have demonstrated that perturbations in early life, especially in the critical developmental window of intrauterine life, have programming effects on health outcomes in later life. A growing body of literature has also demonstrated that prenatal maternal stress (PNMS) can adversely impact a variety of developmental outcomes in the offspring throughout childhood that persist into adulthood $[1,2]$. Epigenetics is considered to be one of the most important mechanisms involved in the long-term effects of in utero stress exposure. In the present review, we summarize the body of human research, exploring the association between fetal experience and epigenetic change, especially DNA methylation. Evidence reported here from research on genes that are associated with intrautrine adversity has shed light on how DNA methylation patterns may mediate biological processes involved in stress regulation. Furthermore, we provide evidence about these associations that have been reported from Project Ice Storm, a human model of the effects of PNMS on subsequent child and adolescent development.

\section{The Fetal Programming Hypothesis}

The fetal programming hypothesis, originally proposed by Barker [3], has been studied intensely in animals [4-9] and has also been applied to human populations [10-15]. It stipulates that behaviors and health outcomes may result from permanent alterations in the structure and function of fetal organs. The primary mechanism believed to be involved in fetal programming of later outcomes is exposure to maternal cortisol. Animal research has shown that elevated maternal cortisol levels negatively impact the developing fetal hypothalamic- 
pituitary-adrenal (HPA) axis resulting in less than optimal outcomes. Stress activates maternal HPA axis functioning and triggers glucocorticoid (GC) secretion that reaches the fetus by transplacental passage. In human study, a linear relationship has been reported between maternal and fetal cortisol levels; relatively small increases in maternal cortisol can double fetal concentrations [16]. Furthermore, stress in the mother not only increases her own circulating cortisol levels but also reduces the activity of the GC barrier enzyme in the placenta, HSD11B2 (11ß-hydroxysteroid dehydrogenase 2, which converts noxious maternal cortisol into inactive cortisone before reaching the fetus), leaving her fetus less well protected [17].

\section{PNMS}

Maternal exposure to a severe stressor, such as environmental pollutants, nutritional factors, psychosocial stress, or maternal depression during pregnancy, can increase the fetus' risk for suboptimal growth in a wide range of somatic systems, and for developing a variety of disorders in adulthood (reviewed in [18-20]). Effects of PNMS on fetal and child development, such as immune function [21], brain development [22], and behavior outcomes [23•], have been largely discussed, although most of the research has been in laboratory animals. In humans, as depression and anxiety disorders are common mental health problems during pregnancy [24, 25], their impact on epigenetic changes has also been widely investigated.

\section{DNA Methylation}

Epigenetic mechanisms involve chromatin structures and their modifications such as DNA methylation [26]. The covalent modification of the DNA molecule itself by methylation [27] at the 5' position of the cytosine ring catalyzed by DNA methyltransferases (DNMT) occurs without changes to the DNA sequence. It is well established that DNA methylation plays a critical role in cellular differentiation during development [28]. DNA methylation is involved in gene regulation mostly by silencing promoter activity, either through interfering with the binding of transcription factors [29] or through recruiting methylated DNA binding proteins (MBD) that recruit histone deacetylases (HDACs) and histone methyltransferases (HMTase) which trigger chromatin inactivation [30].

The impact of epigenetics, especially DNA methylation, on downstream gene expression has been well documented and plays a key role in mediating the effect of intrauterinal environmental factors on offspring health outcoms later in life (reviewed in [31]). A better understanding of epigenetic processes in mediating exposure-phenotype relationships would be beneficial for future human prenatal research. The model of the mediating role of epigenetics in fetal programming is illustrated in Fig. 1.

\section{Environmental Factors and DNA Methylation of Candidate Genes}

The NR3Clgene, coding for the glucocorticoid receptor (GR), is the prime candidate gene for epigenetic influence on outcomes as it plays a key regulator role in HPA axis functioning (see Table 1 for detailed description of candidate gene studies). Oberlander et al. [32] were the first to investigate the association between prenatal exposure to maternal depressive symptoms and the methylation status in the $\mathrm{NR} 3 \mathrm{Cl}$ promoter region in newborn infants. Results showed that increased maternal depressed mood in the third trimester was associated with increased methylation of the NGFI-A binding site in $\mathrm{NR} 3 \mathrm{Cl}$ in the infants, which then predicted increases in their HPA stress reactivity. However, levels of maternal depressed mood and use of serotonin reuptake inhibitor (SSRI) antidepressants during the third trimester were not associated with their own material methylation levels. Another study [38 ${ }^{\circ}$ explored the association between maternal psychological well-being (prenatal depression, state-anxiety, pregnancy-related anxiety, and mother-fetus relationship) and methylation of promoter $1 \mathrm{D}, 1 \mathrm{~B}$, and $1 \mathrm{~F}$ of $N R 3 C 1$ in cord blood at birth. Results indicated that, among the psychological variables, pregnancy-related anxiety was significantly associated with $1 \mathrm{~F}$ methylation, particularly in $\mathrm{CpG}$ site 9 , showing that pregnancy-related anxiety explained $7.8 \%$ of variance in methylation level of this $\mathrm{CpG}$ site.

Mulligan et al. [36] examined the mediating effects of methylation levels of the $\mathrm{NR} 3 \mathrm{Cl}$ promoter on the relationship between prenatal maternal material deprivation (intended to reflect availability of financial resources), daily mundane psychosocial stress, and war-related stress and newborn birth weight in a small cohort (25 mother-newborn dyads) from the Democratic Republic of Congo. Their results revealed a significant negative correlation between these culturally relevant measures of PNMS and newborn birth weight. Furthermore, methylation in $\mathrm{NR} 3 \mathrm{Cl}$ promoter was found to interact with this correlation. In another study of extreme maternal psychosocial stressors derived from the Tutsi genocide [39•], traumatized widows and their 17-18-year-old children were recruited to explore the transgenerational transmission of maternal stress. It was observed that methylation levels of the $\mathrm{NR} 3 \mathrm{Cl}$ exon $1 \mathrm{~F}$ in in utero exposed children were $1.8 \%$ higher than those observed in non-exposed children. A study by Radtke et al. [34] has identified the prenatal period as a critical window for this epigenetic effect. They analyzed the NR3C1 methylation status in 10- to 19-year-old children of mothers who experienced intimate partner violence (IPV) before, during, and after pregnancy. The methylation levels 
Fig. 1 The key role of epigenetic mechanisms in mediating the long-term effects of exposure to intrauterine factors on offspring's health outcomes

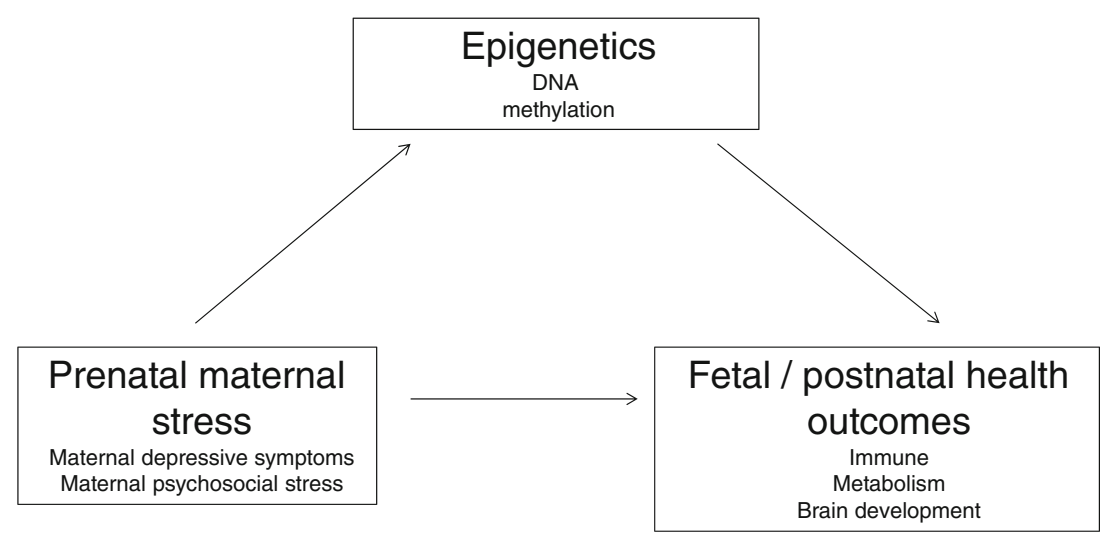

of NR3C1 were significantly higher in children whose mothers experienced IPV while pregnant relative to children whose mothers did not experience IPV during pregnancy. Interestingly, this relationship was not seen when the IPV occurred before or after pregnancy. That such a strong association was observed after more than a decade suggests that transgenerational effects can be maintained and may potentially have lifelong influences on HPA axis via NR3C1 regulation.

Epigenetic effects from other candidate genes have also been investigated. $H S D 11 B 2$, the cortisol catalyzing gene, plays a critical role in the regulation of fetal cortisol exposure. Evidence from animal research suggests that an association exists between prenatal stress and HSD11B2 methylation levels [40]. In humans, the epigenetic role of HSD11B2 was revealed through a study of prenatal socioeconomic adversity by Appleton et al. [37•]. The authors observed that maternal socioeconomic adversity during pregnancy was associated with lower levels of placental HSD11B2 methylation in children, particularly in males. In the same study, the authors investigated whether methylation status of SLC6A4 and of $B D N F$ (brain-derived neurotrophic factor) in neonatal cord blood was associated with maternal depressed mood in the second and third trimesters [33]. Their results showed that increased second (but not third) trimester maternal depressed mood was associated with decreased maternal and infant $S L C 6 A 4$ promoter methylation; the $B D N F$ promoter methylation status in both mothers and infants was not associated with maternal depressed mood in pregnancy.

Potential epigenetic markers believed to play a role in the relationship between maternal depressed mood and birth outcomes have also been investigated [35]. In a study of more than 500 pregnant women which investigated the role of imprinted genes, the authors examined the association between maternal depressed mood during pregnancy and birth weight. Their goal was to determine the extent to which this association was influenced by DNA methylation differences for differentially methylated regions (DMRs) regulating nine imprinted genes (IGF2/H19, DLK1/MEG3, MEST, PEG3,
PEG10/SGCE, NNAT, and PLAGL1). The results indicated that severe maternal depressed mood was associated with a threefold increase in the risk of low birth weight and DNA methylation levels were $2.4 \%$ higher in neonates exposed to severe maternal depressed mood during pregnancy. The authors concluded that MEG3 IGF2 plasticity may play important roles in regulating the association between prenatal maternal depressed mood and low birth weight.

\section{Environmental Factors and Genome-Wide DNA Methylation}

Although many candidate gene studies have shed light on the epigenetic mechanisms of PNMS, genome-wide approaches offer additional insights. Table 2 presents summaries of the human genome-wide studies described in the following sections. For example, Schroeder et al. [41] examined the genome-wide methylation patterns in umbilical cord blood obtained from the neonates of women treated for mood disorders during the perinatal period, and from women without current depression. No significant DNA methylation changes were observed in the cord blood of neonates exposed to maternal depressive symptoms. However, fetal exposure to antidepressants was associated with differential methylation of TNFRSF 21 and CHRNA2 CpG sites, although the average group differences in methylation for these two $\mathrm{CpG}$ sites were less than $3 \%$. These weak findings probably stem from the fact that all women in the control group had previously been diagnosed with a mood disorder and may have had residual symptoms or other vulnerability factors that rendered them more similar to the depressed group than a "supernormal" control group would have been. In another similar study [42] on the effect of antidepressant medication during pregnancy, the authors investigated if different epigenomic patterns can be detected in umbilical cord blood of neonates of mothers with non-medicated depression or anxiety or mothers using selective serotonin reuptake inhibitors (SSRIs) during pregnancy compared to neonates of healthy non-medicated 


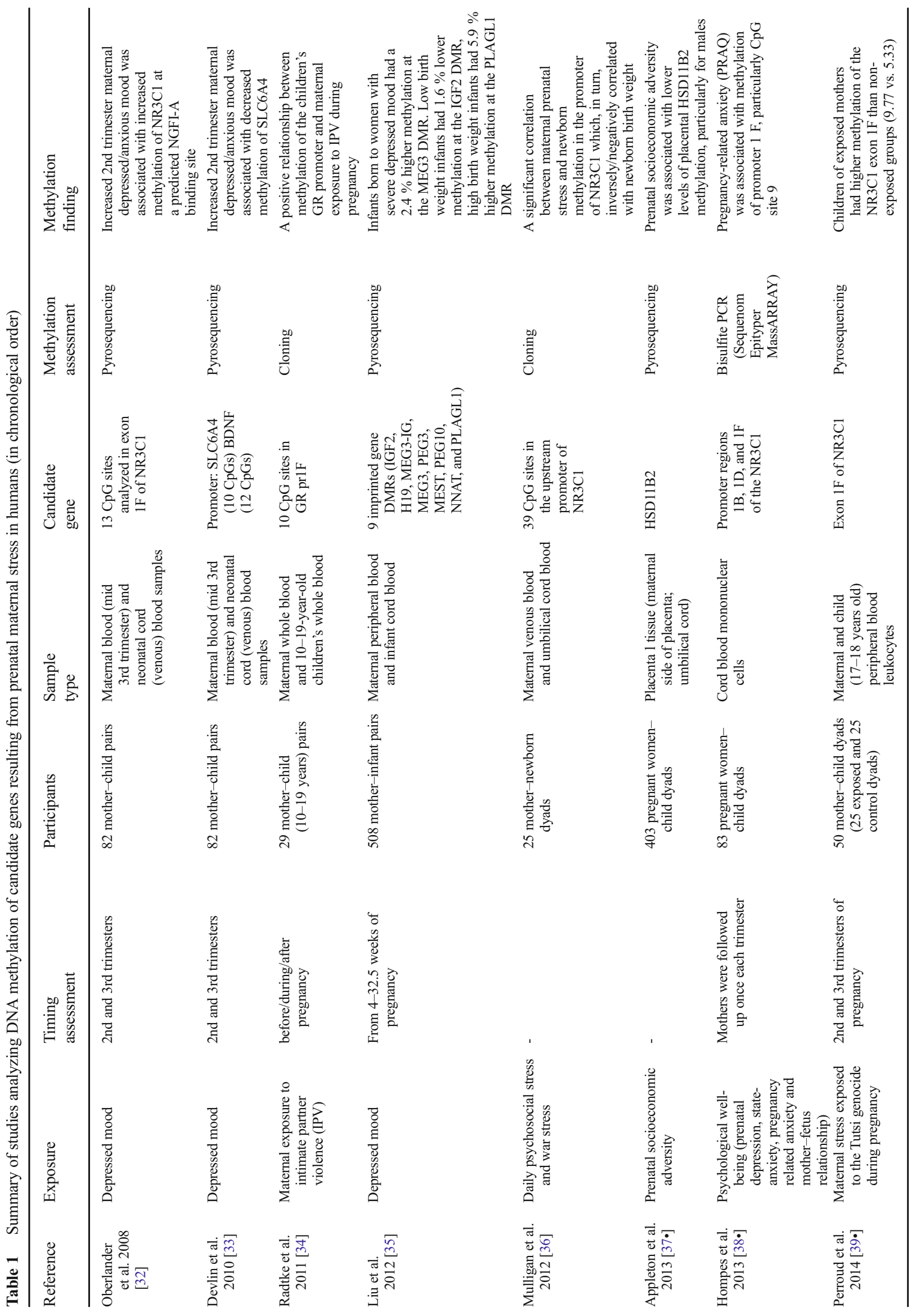




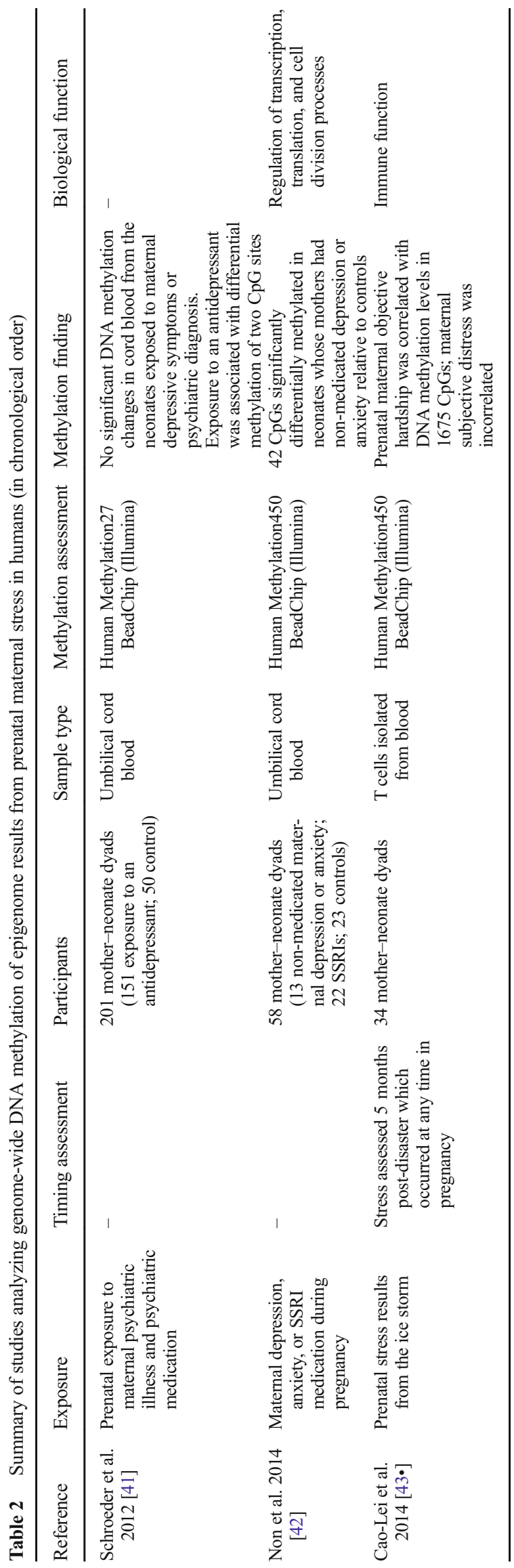

controls. The results indicated that $42 \mathrm{CpG}$ sites were significantly differentially methylated in neonates whose mothers had non-medicated depression or anxiety relative to controls. The largest DNA methylation difference was observed in COL7A1. Furthermore, using a gene ontology analysis, the authors reported that significant clustering of the top genes associated with non-medicated maternal depression/anxiety was related to regulation of transcription, translation, and cell division processes. Interestingly, $\mathrm{CpG}$ methylation differences were not observed in neonates whose mothers had taken SSRIs relative to the controls. Both findings suggest that prenatal exposure to some (but not all) psychotropic medications, maternal psychiatric symptoms, or the combination of the two could impact on offspring outcomes by a mediating effect of DNA methylation.

Taken together, both candidate gene and genome-wide approaches provide evidence supporting the role of epigenetic mechanism in fetal programming.

\section{Natural Disasters as Models of Human PNMS}

Human research cannot match research with laboratory animals for its random assignment of animals to groups and its control of experimental variables. Retrospective psychosocial stress studies [44-48] query mothers directly about life events during the pregnancy and correlate these events to outcomes. However, life event studies are hampered by the rarity of serious events during pregnancy, and by the fact that many events (e.g., marital discord and job loss) may not have been "independent" of the women's own characteristics. Thus, life event studies present challenges to internal validity because many such events are not randomly assigned and characteristics of a woman that increase her risk of experiencing these events (e.g., impulsivity) are likely to be heritable and influence the offspring genetically. Similarly, studies of maternal anxiety or mood in pregnancy find associations with developmental difficulties in children [49-51], but because anxiety is not randomly assigned, it is challenging to disentangle genetic effects from effects of the intrauterine and postnatal environments. Therefore, one approach to circumventing these methodological challenges is to study the effects of exposure to an independent random stressor, such as a natural disaster.

A disaster is any event that "causes disruption exceeding the adjustment capacity of the affected community" [52]. Many disasters act as "natural experiments", distributing hardship in quasi-random fashion such that the degree of objective exposure is independent of personal control or psychosocial circumstances. Disasters also affect large numbers of pregnant women, which is important for obtaining sample sizes needed for advanced analyses. They also have sudden onsets that allow the researcher to accurately time the event in pregnancy, which is important for dating PNMS effects with respect to fetal 
development. Furthermore, disasters allow researchers to distinguish between pregnant women's objective exposure to an event and their subjective distress, since these may not be highly correlated, may have different effects on the fetus, and may have different implications for prevention and intervention. Project Ice Storm is the first human prospective study using a natural disaster to explore the effects of different components of PNMS on developmental outcomes in offspring.

\section{Project Ice Storm}

In January 1998, 5 days of freezing rain collapsed the power grid of southern Quebec leaving three million people without electricity for as long as 45 days in the coldest month of the year. The Canadian Insurance Bureau counts the 1998 ice storm as Canada's worst natural disaster in history. Five months after the ice storm, King recruited 224 women who were pregnant during the crisis. She sent them questionnaires to assess their levels of objective hardship (threat, loss, scope, and change), their cognitive appraisal of the crisis (negative, neutral, positive), and their degree of subjective distress (posttraumatic stress symptoms). The women also provided saliva samples over $24 \mathrm{~h}$ to assess diurnal cortisol. Maternal psychopathology and child development have been monitored since then through questionnaires beginning at 6 months postpartum, and face-to-face assessments beginning at 2 years. Each child assessment includes cognitive, behavioral, motor, and physical assessments. Physical assessments in our protocols have included measures of cardiovascular function (resting heart rate and blood pressure, heart rate variability, stress response), HPA axis (cortisol awakening response, diurnal cortisol, stress reactivity), growth and metabolism (body mass index (BMI), skin folds, glucose challenge), immune function (cytokines, cell populations, history of asthma and allergies), and physical markers (dermatoglyphic asymmetry, brain structure, finger length ratios) (for details, see review in [23•]).

We recently reported changes in genome-wide DNA methylation levels triggered by in utero exposure to the ice storm [43•]. Using blood obtained from a subsample of Project Ice Storm teenagers, we assessed the DNA methylation status in T cells and correlated the severity of the mothers' objective hardship with DNA methylation using Illumina Infinium $450 \mathrm{~K}$. Our analysis revealed that the mothers' objective hardship was significantly correlated with 1675 differentially methylated CpG sites affiliated with 957 genes that are predominantly related to immune function; the mothers' subjective distress from the ice storm was completely uncorrelated with their children's genome-wide methylation profiling in $\mathrm{T}$ cells. In addition, we compared methylation between children whose mothers had a negative cognitive appraisal of the ice storm with children whose mothers had either a neutral or positive appraisal of the ice storm [53]. We found that 2872
$\mathrm{CpG}$ sites were differentially methylated as a function of maternal cognitive appraisal, an even stronger result than that observed with objective stress [43•]. Moreover, there was a significant overlap, but also considerable non-overlap, in the differentially methylated CpGs or genes and biological pathways that were associated with cognitive appraisal and objective PNMS, suggesting that, indeed, different aspects of the maternal stress experience may be operating through distinct, yet overlapping, mechanisms.

Results from this Project Ice Storm study also address the specificity of the results. Of importance, we also demonstrated the comparability of results across $\mathrm{T}$ cells, peripheral blood mononuclear cells (PBMCs), and saliva which provide evidence that the effects of objective PNMS on DNA methylation can be observed in peripheral tissues [43॰].

Project Ice Storm data go beyond the association between an environmental event and DNA methylation levels to show that the epigenetic profile then predicts health outcomes in the offspring. We tested the hypothesis that DNA methylation mediates the effect of PNMS on immune and metabolic outcomes. We selected NF- $\mathrm{kB}$ signaling-related genes (immune) and type 1 and 2 diabetes-related genes (metabolic) to test whether their methylation status mediated the effect of objective and/or subjective PNMS on Th1- and Th2-cytokine production in blood in the $13 \frac{1}{2} 2$-year-old Project Ice Storm children. We found a negative mediation effect of DNA methylation on the relationship between objective PNMS and IFN- $\gamma$ and a positive mediation of the relationship between objective PNMS and IL-2, IL-4, and IL-13. Similarly, DNA methylation of type 1 and 2 diabetes-related genes had a negative mediating effect on BMI and central adiposity. Given that greater objective stress predicts greater BMI and central adiposity, the negative mediation suggests that the alterations in DNA methylation profile are protective, and associated with lower levels of adiposity, rather than an explanatory mechanism for the positive effects of PNMS on obesity [54•]. These data provide evidence supporting the mediating role of DNA methylation on the association between objective aspects of PNMS and child immune and metabolic status.

\section{Conclusions and Discussion}

The prenatal period is a vulnerable time for fetal development in humans. In utero exposure to environmental stress seems to result in long-term epigenome alterations. In this review, we outlined evidence from human prenatal research supporting the view that PNMS could lead to lasting, broad, and functionally organized signatures in DNA methylation which, in turn, could mediate exposure-phenotype associations. We also emphasized the advantage of using disaster-related stress for studying prenatal maternal stress. Although considerable 
discoveries in this field have been made, many challenges still need to be addressed.

Since the seminal finding reported by Weaver et al. [55] that maternal behavior can program the epigenome of the promoter $1_{7}$ of $\mathrm{NR} 3 \mathrm{Cl}$ in rats, this gene has received considerably more attention. Although the critical role of $N R 3 C 1$ in the stress response has been shown to influence fetal development leading to the dysregulation of the infant's HPA axis, the role of other candidate genes in the regulation of the fetal stress response needs further investigation. For example, in the same cohort studied by Oberlander's group, the authors [33] also revealed that the methylation status of SLC6A4 was altered by maternal depressive symptom during pregnancy. In addition, epigenetic reprogramming in response to early adverse environments is not limited to a few candidate genes but is an organized response involving multiple gene networks $[56,57]$. The "genome-wide" approach is atheoretical and exploratory but provides opportunities to inform the researcher of patterns across much of the epigenome. This approach could result in the discovery of new functional gene pathways, leading to new potential mechanistic insights. In this regard, further research should not be limited to selected candidate genes, and it would be benefited by using new approaches with the aim of discovering new biomarkers for health and disease.

Increasing evidence supports the view that stable epigenetic markers could be triggered by exposure to prenatal stress. To date, the Tutsi genocide study provides the most convincing evidence of this stability; NR3C1 methylation changes were still observable in individuals almost 20 years after their mothers were exposed to these traumatic events while pregnant [39॰]. Similarly, epigenetic patterns of $N R 3 C 1$ of children prenatally exposed to the effects of intimate partner violence were detectable 10 to 19 years after birth [34]. In addition, the genome-wide epigenetic signatures of prenatally exposed children were still measurable in immune cells 13 years after the 1998 Quebec ice storm [43•]. These results suggest that continued longitudinal assessment of offsping exposed to PNMS will provide a more comprehensive understanding of the enduring impact of PNMS on the epigenome. The ability of prospective disaster-related PNMS studies to identify the active ingredient in these effects, both maternal objective exposure and cognitive appraisal in the case of Project Ice Storm, is a distinct advantage of this approach.

Environmental programming effects are often sex-specific [58], and there is increasing awareness of possible sexual dimorphisms in environmental epigenetic programming (reviewed in [59]). It was suggested that environmental factors could trigger, in a sex-specific manner, epigenetic biomarkers during developmental windows throughout the individual's life under the control of sex chromosomes and/or sex hormones. Although the sex-specific epigenetic markers related to prenatal adversity have been better investigated in animals, a limited number of studies in humans have examined sex differences in methylation of candidate genes or genomewide level. As mentioned above, the methylation pattern of placental HSD11B2 related to prenatal socioeconomic adversity showed methylation sex specificity, with effects seen particularly in males [37•]. More work is required to identify novel sex-associated CpGs or genes in further studies.

Although epigenetic patterns are considered to be cell- or tissue-specific [28, 60], emerging evidence has also shown that early life stressors, such as social-economic positioning in humans [57] and maternal deprivation in rhesus monkeys [61], are associated with broad DNA methylation signatures in blood [57] and in T cells [61]. Supporting this idea, findings from Project Ice Storm have revealed that the DNA methylation pattern in T cells is comparable with that observed in both PBMCs and saliva samples in children. Since most of the human prenatal research uses neonatal cord blood or placental tissue as biological materials, as such, these studies shared a common limitation of a heterogeneous mixture of different type of cells. Hence, it may be important in future studies to perform detailed analyses of epigenetic status with welldistinguished cell- or tissue-type samples.

It should be stressed that although epigenetic mechanisms, in particular DNA methylation, have been postulated to play a mediating role through which PNMS may have important lifespan health consequences, only a few studies have conducted empirical tests of these effects using mediation analyses. Using bootstrapping analysis, we found that objective hardship experienced by the pregnant women during the ice storm had a large impact on genome-wide DNA methylation level, which, in turn, mediated the effect on immune and metabolic outcomes. However, more replication is warranted in this field.

Although standard bisulfite-based sequencing approaches, such as pyrosequencing, are powerful methods to explore DNA methylation, it should be noted that they cannot distinguish between 5-methyl-cytosine $(5-\mathrm{mC})$ and 5hydroxymethyl-cytosine $(5-\mathrm{hmC})$ which is considered as the "sixth base". Recently, hydroxymethylation has been gaining more attention and revealing a new layer of epigenetic regulation. However, few studies have attempted to link hydroxymethylated DNA biomarkers to exposure to prenatal maternal stress.

A primary interest in epigenetic research is to find relevant epigenetic biomarkers. However, the magnitude of changes in DNA methylation of many biomarkers discussed above are very modest (rarely exceeds $10 \%$ ). It remains unclear of how these modest DNA methylation changes actually affect functional downstream factors which influence phenotypes. An explanation could be that the observed magnitude of change was somewhat deflated due to confounding factors. It is also possible that the association between DNA methylation and gene expression might be less straightforward or other 
additional layers of epigenetic regulation, above and beyond DNA methylation, are required to regulate gene expression. Regardless, in order to gain better insight into the biological function of these modest DNA methylation changes, gene expression experiments are required to determine the functional repercussions of observed alterations in DNA methylation due to prenatal maternal stress exposure. However, only a few studies $[39 \bullet, 62]$ have examined gene expression by accessing mRNA and/or protein levels in relation to epigenetic modifications. Moreover, it is also important to identify whether upstream factors/pathways could direct differential DNA methylation to a small subset of CpGs as biomarkers in the epigenome, and furthermore, questions remain to be explored in understanding of the potential underlying mechamisms such as loss of methylation due to transcriptional activation or transient/sustained inhibition of DNMT containing complex recruitment. Taken together, there is a need for follow-up studies to more precisely address these issues.

In summary, we have reviewed evidence from human studies that prenatal maternal stress can have important epigenetic consequences in genes that are associated with environmental adversity, and that DNA methylation profiles appear to have long-term consequences for adaptation and development in the unborn child.

\section{Compliance with Ethical Standards}

Conflict of Interest Lei Cao-Lei, David P. Laplante, and Suzanne King report grants and personal fees from CIHR, during the conduct of the study.

Human and Animal Rights and Informed Consent This article does not contain any studies with human or animal subjects performed by any of the authors.

\section{References}

Papers of particular interest, published recently, have been highlighted as:

- Of importance

1. Eriksson JG. Early programming of later health and disease: factors acting during prenatal life might have lifelong consequences. Diabetes. 2010;59:2349-50.

2. Beydoun H, Saftlas AF. Physical and mental health outcomes of prenatal maternal stress in human and animal studies: a review of recent evidence. Paediatr Perinat Epidemiol. 2008;22:438-66.

3. Barker DJ. The fetal and infant origins of adult disease. Br Med J. 1990;301:1111.

4. McArdle HJ, Andersen HS, Jones H, Gambling L. Fetal programming: causes and consequences as revealed by studies of dietary manipulation in rates - a review. Placenta. 2006;27:S56-60.
5. Schneider ML, Moore CF. Effect of prenatal stress on development: a nonhuman primate model. In: Nelson C, editor. Minnesota symposium on child psychology. Hillsdale, NJ: Lawrence Erlbaum; 2000. p. 201-43.

6. Son GH, Chung S, Geum D, Kang SS, Choi WS, Kim K, et al. Hyperactivity and alteration of the midbrain dopaminergic system in maternally stressed male mice offsrping. Biochem Biophys Res Commun. 2007;352:823-9.

7. Weinstock M. Alterations induced by gestational stress in brain morphology and behaviour of the offspring. Prog Neurobiol. 2001;65:427-51.

8. Welberg LAM, Seck1 JR. Prenatal stress, glucocorticoids and the programming of the brain. J Neuroendocrinol. 2001;13:113-28.

9. Kapoor A, Petropoulos S, Matthews SG. Fetal programming of hypothalamic-pituitary-adrenal (HPA) axis function and behavior by synthetic glucocorticoids. Brain Res Rev. 2008;57:586-95.

10. Barker DJ. The fetal and infant origins of disease. Eur J Clin Invest. 1995;25:457-63.

11. Barker DJ, Martyn CN. The fetal origins of hypertension. Adv Nephrol Necker Hosp. 1997;26:65-72.

12. O'Connor TG, Heron J, Golding J, Beveridge M, Glover V. Maternal antenatal anxiety and children's behavioural/emotional problems at 4 years. Br J Psychiatry. 2002;180:502-8.

13. O'Connor TG, Heron J, Golding J, Glover V, Team AS. Maternal antenatal anxiety and behavioural/emtional problems in children: a test of a programming hypothesis. J Child Psychol Psychiatry. 2003;44:1025-36.

14. Oken E, Gillman MW. Fetal origins of obesity. Obes Res. 2003;11: 496-506.

15. Egliston KA, McMahon C, Austin MP. Stress in pregnancy and infant HPA axis function: conceptual and methodological issues relating to the use of salivary cortisol as an outcome measure. Psychoneuroendocrinology. 2007;32:1-13.

16. Gitau R, Cameron A, Fisk NM, Glover V. Fetal exposure to maternal cortisol. Lancet. 1998;352:707-8.

17. Avishai-Eliner S, Brunson KL, Sandman CA, Baram TZ. Stressedout, or in (utero)? Trends Neurosci. 2002;25:518-24.

18. Perera F, Herbstman J. Prenatal environmental exposures, epigenetics, and disease. Reprod Toxicol. 2011;31:363-73.

19. Szyf M. DNA methylation, behavior and early life adversity. J Genet Genomics. 2013;40:331-8.

20. Billack B, Serio R, Silva I, Kinsley CH. Epigenetic changes brought about by perinatal stressors: a brief review of the literature. J Pharmacol Toxicol Methods. 2012;66:221-31.

21. Veru F, Laplante DP, Luheshi G, King S. Prenatal maternal stress exposure and immune function in the offspring. Stress. 2014;17: 133-48.

22. Charil A, Laplante DP, Vaillancourt C, King S. Prenatal stress and brain development. Brain Res Rev. 2010;65:56-79.

23. King S, Dancause K, Turcotte-Tremblay A-M, Veru F, Laplante DP. Using natural disasters to study the effects of prenatal maternal stress on child health and development. Birth Defects Res Part C: Embryo Today: Rev. 2012;96:273-88. This review summarizes the findings of Project Ice Storm, the largest longitudinal human prospective study using a natural disaster, on exploring the effects of disaster ice storm on developmental outcomes in offspring.

24. Bennett HA, Einarson A, Taddio A, Koren G, Einarson TR. Prevalence of depression during pregnancy: systematic review. Obstet Gynecol. 2004;103:698-709.

25. Ross LE, McLean LM. Anxiety disorders during pregnancy and the postpartum period: a systematic review. J Clin Psychiatry. 2006;67: 1285-98.

26. Jenuwein T. Re-SET-ting heterochromatin by histone methyltransferases. Trends Cell Biol. 2001;11:266-73. 
27. Hotchkiss RD. The quantitative separation of purines, pyrimidines, and nucleosides by paper chromatography. J Biol Chem. 1948;175: 315-32.

28. Razin A, Szyf M. DNA methylation patterns. Formation and function. Biochim Biophys Acta. 1984;782:331-42.

29. Barrelet L, Ferrero F, Szigethy L, Giddey C, Pellizzer G. Expressed emotion and first-admission schizophrenia: nine-month follow-up in a French cultural environment. Br J Psychiatry. 1990;156:35762.

30. Nan X, Ng HH, Johnson CA, Laherty CD, Turner BM, Eisenman $\mathrm{RN}$, et al. Transcriptional repression by the methyl-CpG-binding protein $\mathrm{MeCP} 2$ involves a histone deacetylase complex [see comments]. Nature. 1998;393:386-9.

31. Wadhwa PD, Buss C, Entringer S, Swanson JM. Developmental origins of health and disease: brief history of the approach and current focus on epigenetic mechanisms. Semin Reprod Med. 2009;27:358-68.

32. Oberlander TF, Weinberg J, Papsdorf M, Grunau R, Misri S, Devlin AM. Prenatal exposure to maternal depression, neonatal methylation of human glucocorticoid receptor gene (NR3C1) and infant cortisol stress responses. Epigenetics : Off J DNA Methylation Soc. 2008;3:97-106

33. Devlin AM, Brain U, Austin J, Oberlander TF. Prenatal exposure to maternal depressed mood and the MTHFR C677T variant affect SLC6A4 methylation in infants at birth. PLoS One. 2010;5:e12201.

34. Radtke KM, Ruf M, Gunter HM, Dohrmann K, Schauer M, Meyer A, et al. Transgenerational impact of intimate partner violence on methylation in the promoter of the glucocorticoid receptor. Transl Psych. 2011;1:e21.

35. Liu Y, Murphy SK, Murtha AP, Fuemmeler BF, Schildkraut J, Huang Z, et al. Depression in pregnancy, infant birth weight and DNA methylation of imprint regulatory elements. Epigenetics. 2012;7:735-46.

36. Mulligan CJ, D'Errico NC, Stees J, Hughes DA. Methylation changes at $\mathrm{NR} 3 \mathrm{C} 1$ in newborns associate with maternal prenatal stress exposure and newborn birth weight. Epigenetics Off J DNA Methylation Soc. 2012;7:853-7.

37. Appleton AA, Armstrong DA, Lesseur C, Lee J, Padbury JF, Lester BM, et al. Patterning in placental 11-B hydroxysteroid dehydrogenase methylation according to prenatal socioeconomic adversity. PLoS ONE. 2013;8:e74691. This paper reportes 11-B hydroxysteroid dehydrogenase as a novel biomarker that was triggered by prenatal socioeconomice adverstiy.

38. Hompes T, Izzi B, Gellens E, Morreels M, Fieuws S, Pexsters A, et al. Investigating the influence of maternal cortisol and emotional state during pregnancy on the DNA methylation status of the glucocorticoid receptor gene $(\mathrm{NR} 3 \mathrm{C} 1)$ promoter region in cord blood. $\mathrm{J}$ Psychiatr Res. 2013;47:880-91. In this study the psychological well-being was explored by accessing the prenatal depression, state-anxiety, pregnancy related anxiety and mother-fetus relationship. Most significant findings concerned $1 \mathrm{~F}$ which was found to be significantly associated with maternal emotional wellbeing.

39. Perroud N, Rutembesa E, Paoloni-Giacobino A, Mutabaruka J, Mutesa L, Stenz L, et al. The Tutsi genocide and transgenerational transmission of maternal stress: epigenetics and biology of the HPA axis. World J Biol Psych: Off J World Fed Soc Biol Psych. 2014;15: 334-45. This paper uses an extreme maternal psychosocial stressors derived from Tutsi genocide to explore the transgenerational transmission of maternal stress on NR3C1.

40. Jensen Pena C, Monk C, Champagne FA. Epigenetic effects of prenatal stress on 11 beta-hydroxysteroid dehydrogenase- 2 in the placenta and fetal brain. PLoS ONE. 2012;7:e39791.

41. Schroeder JW, Smith AK, Brennan PA, Conneely KN, Kilaru V, Knight BT, et al. DNA methylation in neonates born to women receiving psychiatric care. Epigenetics. 2012;7:409-14.
42. Non AL, Binder AM, Kubzansky LD, Michels KB. Genome-wide DNA methylation in neonates exposed to maternal depression, anxiety, or SSRI medication during pregnancy. Epigenetics. 2014;9: 964-72.

43. Cao-Lei L, Massart R, Suderman M, Machnes Z, Laplante D, Szyf M, et al. DNA methylation signatures of prenatal maternal objective stress exposure to a natural disaster: project Ice storm. PLoS ONE. 2014;9: e107653. This paper demonstrates genome-wide DNA methylation profiling in $\mathbf{T}$ cells obtained from youth who were exposed in utero to varying levels of disaster-maternal stress. The analysis revealed differentially methylated CGs affiliated with genes predominantly related to immune function that were correlated with the degree of objective hardship. The DNA methylation pattern were comparable in $T$ cells, PBMCs and saliva samples.

44. Barrett ES, Parlett LE, Sathyanarayana S, Liu F, Redmon JB, Wang $\mathrm{C}$, et al. Prenatal exposure to stressful life events is associated with masculinized anogenital distance (AGD) in female infants. Physiol Behav. 2013;114-115:14-20.

45. Bergman K, Sarkar P, O'Connor TG, Modi N, Glover V. Maternal stress during pregnancy predicts cognitive ability and fearfulness in infancy. J Am Acad Child Adolesc Psychiatry. 2007;46:1454-63.

46. Entringer S, Kumsta R, Hellhammer DH, Wadhwa PD, Wust S. Prenatal exposure to maternal psychosocial stress and HPA axis regulation in young adults. Horm Behav. 2009;55:292-8.

47. Gutteling BM, de Weerth C, Zandbelt N, Mulder EJH, Visser GHA, Buitelaar JK. Does maternal prenatal stress adversely affect the child's learning and memory at age six? J Abnorm Child Psychol. 2006;34:789-98.

48. Mohler E, Parzer P, Brunner R, Wiebel A, Resch F. Emotional stress in pregnancy predicts human infant reactivity. Early Hum Dev. 2006;82:731-7.

49. Mennes M, Stiers P, Lagae L, Van den Bergh BRH. Long-term cognitive sequelae of antenatal maternal anxiety: involvement of the orbitofrontal cortex. Neurosci Biobehav Rev. 2006;30:1078-86.

50. Van den Bergh BRH, Mennes M, Stevens V, Van der Meere J, Borger N, Stiers P, et al. ADHD deficits as measured in adolescent boys with a continuous performance task is related to antenatal maternal anxiety. Pediatr Res. 2006;59:78-82.

51. Van den Bergh BR, Van Calster B, Smits T, Van Huffel S, Lagae L. Antenatal maternal anxiety is related to HPA-axis dysregulation and self-reported depressive symptoms in adolescence: a prospective study on the fetal origins of depressed mood. Neuropsychopharmacology. 2008;33:536-45.

52. Lechat MF. Disasters and public health. Bull World Health Organ. 1979;57:11-7.

53. Cao-Lei L, Elgbeili G, Massart R, Laplante DP, Szyf M, King S (2015): Pregnant women's cognitive appraisal from a natural disaster affects DNA methylation in their children 13 years later: Project Ice Storm. Transl Psych 5.

54. Cao-Lei L, Dancause KN, Elgbeili G, Massart R, Szyf M, Liu A, et al. DNA methylation mediates the impact of exposure to prenatal maternal stress on BMI and central adiposity in children at age 13(1/ 2) years: Project Ice Storm. Epigenetics. 2015;10:749-61. This study demonstrates that disaster-related prenatal maternal stress was related to central adiposity and BMI in children at age of $131 / 2$. In addition, the methylation levels of genes selected from established diabetes genes in the type 1 and 2 diabetes mellitus pathways showed significant negative mediation of the effect of objective hardship experienced by the mother during pregnancy on central adiposity and BMI.

55. Weaver IC, Cervoni N, Champagne FA, D'Alessio AC, Sharma S, Seckl JR, et al. Epigenetic programming by maternal behavior. Nat Neurosci. 2004;7:847-54.

56. McGowan PO, Suderman M, Sasaki A, Huang TC, Hallett M, Meaney MJ, et al. Broad epigenetic signature of maternal care in the brain of adult rats. PLoS ONE. 2011;6:e14739. 
57. Borghol N, Suderman M, McArdle W, Racine A, Hallett M, Pembrey M, et al. Associations with early-life socio-economic position in adult DNA methylation. Int J Epidemiol. 2012;41:62-74.

58. Gilbert JS, Nijland MJ. Sex differences in the developmental origins of hypertension and cardiorenal disease. Am J Physiol Regul Integr Comp Physiol. 2008;295:R1941-52.

59. Gabory A, Attig L, Junien C. Sexual dimorphism in environmental epigenetic programming. Mol Cell Endocrinol. 2009;304:8-18.

60. Benvenisty N, Szyf M, Mencher D, Razin A, Reshef L. Tissuespecific hypomethylation and expression of rat phosphoenolpyruvate carboxykinase gene induced by in vivo treatment of fetuses and neonates with 5-azacytidine. Biochemistry (Mosc). 1985;24: 5015-9.

61. Provencal N, Suderman MJ, Guillemin C, Massart R, Ruggiero A, Wang $\mathrm{D}$, et al. The signature of maternal rearing in the methylome in rhesus macaque prefrontal cortex and T cells. J Neurosci. 2012;32:15626-42.

62. Hogg K, Blair JD, McFadden DE, von Dadelszen P, Robinson WP. Early onset pre-eclampsia is associated with altered DNA methylation of cortisol-signalling and steroidogenic genes in the placenta. PLoS ONE. 2013;8:e62969. 